\title{
ATIVIDADE RESIDUAL DE IMAZAQUIN E ALACHLOR+ATRAZINE PARA PLANTIO SEQÜENCIAL DE CANOLA
}

\author{
CARRYOVER OF IMAZAQUIN AND ALACHLOR+ATRAZINE \\ TO THE SUCCESSIONAL CANOLA CROP
}

\author{
Rubem Silvério de Oliveira Júnior ${ }^{1}$
}

RESUMO

No presente trabalho, avaliou-se o efeito residual dos herbicidas imazaquin (150 e $300 \mathrm{~g}$ de equivalente ácido $\left.\mathrm{ha}^{-1}\right)$ e alachlor+atrazine $\left(1430+1430\right.$ e $\left.2860+2860 \mathrm{~g} \mathrm{ha}^{-1}\right)$, aplicados respectivamente em soja e milho, visando a determinar o intervalo de tempo mínimo entre a utilização desses herbicidas e a semeadura de canola em rotação após essas culturas. Amostras de solo foram coletadas aos 15, 30, 60, 90 e 120 dias após a aplicação (DAA) dos herbicidas no campo e dez sementes de canola foram semeadas em cada vaso. A germinação da canola foi reduzida até 30DAA de imazaquin na dose recomendada (150g de equivalente ácido $\mathrm{ha}^{-1}$ ) e até 60DAA no dobro da dose recomendada. Em relação ao crescimento das plantas, o imazaquin reduziu em 30 e 32,5\% (respectivamente para as doses de 150 e $300 \mathrm{~g}$ de equivalente ácido $\mathrm{ha}^{-1}$ ) a produção de biomassa nas plantas crescidas em amostras de solo coletadas aos 15DAA. No entanto, a partir dos 30DAA, não houve nenhum sinal de fitotoxicidade nas plantas. Para alachlor+atrazine, os efeitos sobre a germinação e crescimento foram mais intensos nas amostras coletadas até os 30DAA, mas os sintomas de fitotoxicidade decresceram gradativamente e, a partir dos 90DAA, não se observou nenhum efeito negativo sobre as características avaliadas. Os resultados obtidos indicam que a semeadura da canola no campo pode ser feita 90DAA de imazaquin e alachlor+atrazine, sem que haja prejuizos na emergência ou no desenvolvimento da cultura por efeito de resíduos desses herbicidas no solo.

Palavras-chave: Brassica napus, herbicidas, persistência, resíduos.

\section{SUMMARY}

The objective of this work was to evaluate the carryover potential of the herbicides imazaquin (150 and $300 \mathrm{~g}$ eq. acid $\left.h a^{-1}\right)$ and alachlor+atrazine $(1430+1430$ and
$2860+2860 \mathrm{~g} \mathrm{ha}^{-1}$ ), applied respectively to soybeans and corn crops, aiming at determining the smallest period of time between utilization of these products and canola planting date succeding these crops. Soil samples were collected at 15, 30, 60, 90 and 120 days after application (DAA) of the herbicides on field and ten canola seeds were sown in each pot. Canola germination was reduced up to 30DAA of imazaquin applied at recommended rate $\left(150 \mathrm{~g}\right.$ eq. acid $\left.\mathrm{ha}^{-1}\right)$ and up to 60DAA at double the recommended rate. In relation to plant growth, imazaquin inhibited in 30 and $32.5 \%$ (rates of 150 and 300eq. acid ha $\mathrm{a}^{-1}$, respectively) canola shoot dry biomass production, in soil samples collected at 15DAA. Nevertheless, after 30DAA, no visual symptoms of phytotoxicity were shown. For alachlor+atrazine, the effects on germination and growth were more intense in samples collected up to 30DAA, but phytotoxicity symptoms decreased gradually and after 90DAA no negative effects were observed for the parameters evaluated. The results suggest that canola can be planted 90 days after spraying imazaquin and alachlor+atrazine, with no damage to emergence or crop development derived from residues of these herbicides present in soil.

Key words: Brassica napus, herbicides, persistence, residues.

\section{INTRODUÇÃO}

A canola (Brassica napus var oleifera ou Brassica campestris var oleifera) é uma seleção genotipicamente modificada da colza, pertencente à família Brassicaceae (crucíferas). É uma planta anual, de porte ereto, atingindo até 1,60 a $1,70 \mathrm{~m}$ de altura. É considerada uma das alternativas mais promissoras para o cultivo de inverno, após as cultu-

\footnotetext{
${ }^{1}$ Engenheiro Agrônomo, Doutor, Professor Adjunto do Departamento de Agronomia da Universidade Estadual de Maringá. Av. Colombo, 5790, 87020-900 ,Maringá, PR. E-mail: rsojunior@uem.br.
} 
ras da soja ou do milho no verão. O seu cultivo tem como principal objetivo o aproveitamento da semente, a qual contém $27 \%$ de proteínas e 40 a $50 \%$ de óleo (SILVA et al., 1981).

Imazaquin e alachlor+atrazine são herbicidas recomendados para o controle de plantas daninhas nas culturas de soja e milho, respectivamente, e estão entre os produtos mais utilizados nessas culturas na safra de verão, em função de sua comprovada eficiência e seletividade. Ambos são aplicados diretamente ao solo e apresentam efeito residual, o qual proporciona o controle das plantas daninhas por período mais prolongado. Entretanto, a residualidade dos herbicidas pode acarretar problemas de fitotoxicidade nas culturas que são semeadas em rotação, uma vez que já foi demonstrada a sensibilidade da canola a herbicidas aplicados ao solo (OLIVEIRA JR. et al., 1994).

Devido aos muitos fatores envolvidos, não é possível predizer o tempo exato que é requerido para a dissipação de um herbicida aplicado ao solo sob um conjunto particular de condições. A meia vida é um parâmetro comum usado para comparar a persistência de herbicidas no solo. Alachlor e atrazine possuem meia vida no solo estimada em 15 dias (FLURY, 1996) e 55 dias (NAKAGAWA et al., 1995), respectivamente. Sabe-se que a degradação desses compostos no solo é influenciada pela temperatura e conteúdo de água no solo (BOWMAN, 1989; WALKER et al., 1992). O imazaquin possui meia vida média no solo de 60 dias (AHRENS, 1994), mas seu efeito residual ainda pode ser observado, em alguns casos, até 90 a 180 dias após a aplicação (DAA) do herbicida no campo (SILVA et al., 1995; NOVO et al., 1997; SILVA et al., 1997; SILVA et al., 1998). Trabalhos anteriores têm evidenciado que resíduos de imazaquin podem causar efeitos fitotóxicos em culturas semeadas em sucessão à soja tratada com esse herbicida. JOHNSON \& TALBERT (1996), ao avaliarem o efeito residual de imazaquin, demonstraram que resíduos desse herbicida no solo causaram toxicidade às culturas de melão, pepino, girassol e mostarda, até quando semeadas aos 112DAA. Em trabalhos realizados durante três anos consecutivos, WALSH et al. (1993) avaliaram os efeitos residuais no solo de imazaquin, utilizado na cultura da soja, sobre as culturas sucessivas de trigo, milho, sorgo e algodão. Constataram que o imazaquin, na maior dose, causou injúria em algodão, sem, no entanto, afetar a produtividade. RENNER et al. (1988) também detectaram prejuízos para o crescimento e produtividade da cultura do milho plantada após a aplicação de imazaquin em soja.

Este trabalho teve como objetivo avaliar o período de efeito residual dos herbicidas imazaquin e alachlor+atrazine aplicados, respectivamente, às culturas de soja e milho, para determinar o intervalo de tempo mínimo entre a utilização desses herbicidas e a semeadura de canola em rotação após essas culturas.

\section{MATERIAL E MÉTODOS}

Este trabalho foi desenvolvido nas localidade de Iguatemi, município de Maringá, e Cianorte, $\mathrm{PR}$, em solos cujas características químicas encontram-se na tabela 1. Parcelas de $20 \times 30 \mathrm{~m}$ foram conduzidas no campo num delineamento em blocos casualizados, com seis tratamentos e quatro repetições. Os tratamentos foram dispostos num modelo hierárquico, no qual as parcelas, nas quais foi semeada a soja, receberam doses de imazaquin $(0,150 \mathrm{e}$ $300 \mathrm{~g}$ de equivalente ácido $\left.\mathrm{ha}^{-1}\right)$, correspondentes à dose normal recomendada $(\mathrm{X})$ e ao dobro da dose recomendada $(2 \mathrm{X})$, e as parcelas nas quais foi semeado o milho foram aplicadas doses da mistura formulada alachlor+atrazine, nas doses de $0,1430+1430$ (X) e $2860+2860 \mathrm{~g} \mathrm{ha}^{-1}$ (2X). Os herbicidas foram aplicados em pré-emergência, com solo úmido, um dia após a semeadura das culturas. Utilizou-se um pulverizador de barra, equipado com 20 bicos leque 8003, espaçados entre si de $0,50 \mathrm{~m}$, com pressão constante de $276 \mathrm{kPa}$, calibrado para proporcionar um volume de calda de $3001 \mathrm{ha}^{-1}$.

\begin{tabular}{|c|c|c|c|c|c|c|c|c|c|c|}
\hline \multirow[t]{2}{*}{ Local } & \multicolumn{2}{|c|}{$\mathrm{pH}$} & \multirow[t]{2}{*}{$\mathrm{Al}^{3+}$} & \multirow[t]{2}{*}{$\mathrm{H}^{+}+\mathrm{Al}^{3+}$} & \multirow[t]{2}{*}{$\mathrm{Ca}^{+2}+\mathrm{Mg}^{+2}$} & \multirow[t]{2}{*}{$\mathrm{Ca}^{+2}$} & \multirow[t]{2}{*}{$\mathrm{K}^{+}$} & \multirow[t]{2}{*}{$\mathrm{P}$} & \multirow[t]{2}{*}{$\mathrm{C}$} & \multirow[t]{2}{*}{ Textura } \\
\hline & $\mathrm{CaCl}_{2}$ & $\mathrm{H}_{2} \mathrm{O}$ & & & & & & & & \\
\hline & & & $\ldots$ & $\ldots$ & $\mathrm{cmol}_{\mathrm{c}} \mathrm{dm}^{-3}$ & & - & $\mathrm{mg} \mathrm{dm}^{-3}$ & $\%$ & \\
\hline Iguatemi & 5,2 & 5,8 & 0 & 4,28 & 6,52 & 5,56 & 0,58 & 1 & 1,48 & média \\
\hline Cianorte & 4,1 & 4,7 & 1,10 & 3,26 & 3,85 & 2,48 & 0,63 & 2 & 1,88 & argilosa \\
\hline
\end{tabular}


A semeadura foi realizada em 27 de outubro de 1993, utilizando-se a cultivar BR-16 para a soja e o híbrido AG-4011 para o milho. A soja foi semeada no espaçamento de $0,50 \mathrm{~m}$ entre linhas e $0,05 \mathrm{~m}$ entre plantas dentro das linhas. O milho foi semeado no espaçamento de $0,90 \mathrm{~m}$ entre linhas e $0,2 \mathrm{~m}$ entre plantas na mesma linha. As culturas receberam os tratos culturais convencionais, segundo as recomendações da EMBRAPA (1993) e MELHORANÇA et al. (1997).

Visando a avaliar, em casa de vegetação, o efeito dos resíduos desses herbicidas para a cultura da canola, amostras compostas de solo foram retiradas da profundidade de $0-10 \mathrm{~cm}$ em cada parcela tratada no campo. Para compor cada amostra, foram feitas 15 subamostras por parcela, as quais foram misturadas e homogeneizadas. Após a coleta, as amostras foram secas ao ar e peneiradas $(5 \mathrm{~mm})$. Do volume total da amostra, foram retiradas oito subamostras para compor o experimento em casa de vegetação. Essas subamostras foram colocadas em vasos de plástico de $300 \mathrm{~cm}^{3}$ de capacidade, nos quais foram semeados 10 sementes de canola (cv. Iciola 41 ), a uma profundidade média de $0,5 \mathrm{~cm}$. Os vasos foram mantidos em casa de vegetação, período durante o qual os vasos receberam duas irrigações diárias. Quinze dias depois da germinação, foram feitas as contagens do número de plantas vivas. Após a contagem, a parte aérea das plantas foi coletada, procedendo-se à secagem do material em estufa de ventilação forçada, a $72^{\circ} \mathrm{C}$ por 24 horas, para determinação da massa seca.

Em casa de vegetação, utilizou-se um delineamento inteiramente casualizado, com oito repetições. Para cada local coletado e herbicida utilizado, os tratamentos foram combinados num esquema fatorial, com três doses $(0, \mathrm{X}$ e $2 \mathrm{X})$ e seis épocas de coleta das amostras $(0,15,30,60,90$ e 120DAA). Posteriormente, foi feita a análise conjunta dos dois locais, para cada herbicida. Os dados foram submetidos à análise de variância e a época de coleta foi estudada utilizando modelos de regressão, utilizando-se procedimentos do SAEG (SAEG, 1997). Uma vez que não foi possível estabelecer modelos com ajustes satisfatórios aos dados, parâmetros de estatística descritiva (média e desvio-padrão) foram utilizados na apresentação e discussão dos dados.

Visando a eliminar a influência do ambiente nas épocas de condução dos ensaios em casa de vegetação, os dados de germinação e produção de biomasssa foram transformados em valores percentuais em relação aos tratamentos sem herbicida, adotando-se para este tratamento o valor referencial de $100 \%$ para cada época.

Um dia após a colheita da soja e do milho, a área experimental foi arada e gradeada, semandose, em seguida, a canola, conforme as recomendações técnicas para a cultura (SANTOS et al., 1990; SEMINÁRIO..., 1992). Seguindo-se a mesma distribuição espacial e delineamento do experimento realizado para coleta das amostras de solo, foram avaliados o estande da cultura (20 dias após a semeadura) e o rendimento de grãos.

\section{RESULTADOS E DISCUSSÃO}

Nenhuma interação entre herbicidas e locais foi significativa, de modo que os resultados apresentados representam a média dos dois locais. Não ocorreu germinação da canola nas amostras de solo coletadas no dia da aplicação dos herbicidas, fato pelo qual a época de coleta referente a essa data não foi apresentada nas figuras 1 e 2 . A avaliação da germinação da canola nas amostras de solo coletadas após a aplicação de imazaquin evidenciou uma redução significativa na emergência de plantas até 30DAA para a dose recomendada e até 60DAA para o dobro da dose recomendada (Figura 1). Em relação ao crescimento das plantas, observou-se que o imazaquin aplicado até no dobro da dose recomendada reduziu em cerca de $30 \%$ a produção de massa seca da parte aérea das plantas de canola, quando as amostras de solo foram coletadas aos 15DAA (Figura 1). No entanto, não foram observados efeitos significativos de doses nas demais épocas de amostragem de solo, ou seja, a partir das amostras coletadas aos 30DAA, não houve efeito sobre a produção de massa seca da parte aérea de canola. É possível que a redução do número de plantas emergidas tenha proporcionado o maior crescimento relativo das plantas sobreviventes. Tem sido atribuída à canola certa plasticidade morfológica (SANTOS et al., 1990), sendo que o efeito de uma menor densidade de plantas pode ser compensado pelo aumento da produtividade individual das plantas.

Em plantas semeadas aos 15DAA, foram observados sintomas de arroxeamento nas primeiras folhas, logo após a emergência. Esses sintomas são típicos em plantas exibindo sintomas de fitotoxicidade de resíduos de herbicidas do grupo das imidazolinonas (VIDAL, 1997). 


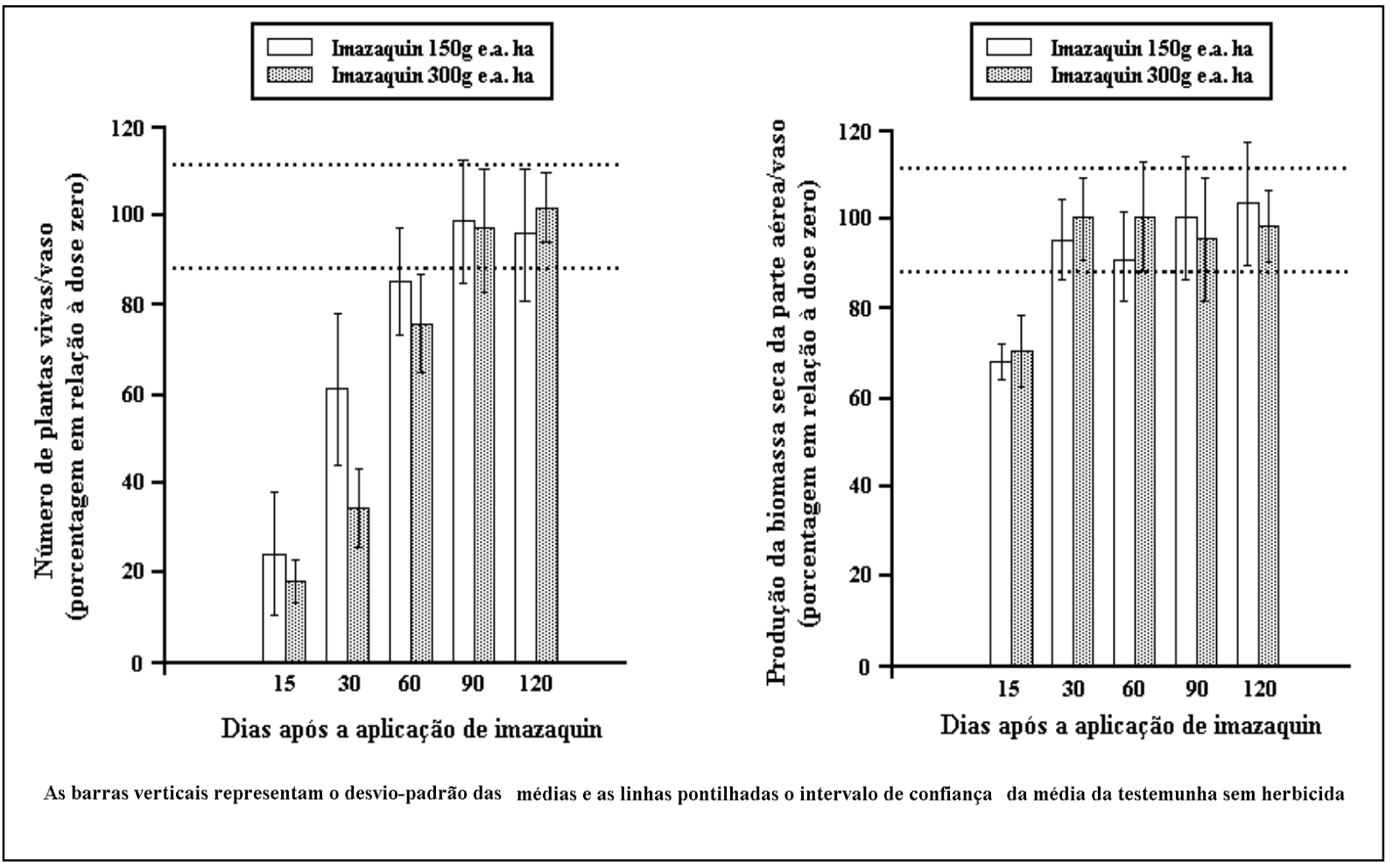

Figura 1 - Efeito de duas doses de imazaquin sobre o número de plantas vivas e peso da massa seca da parte aérea das plantas de canola semeadas em amostras de solo coletadas em diferentes épocas após a aplicação dos herbicidas no campo.

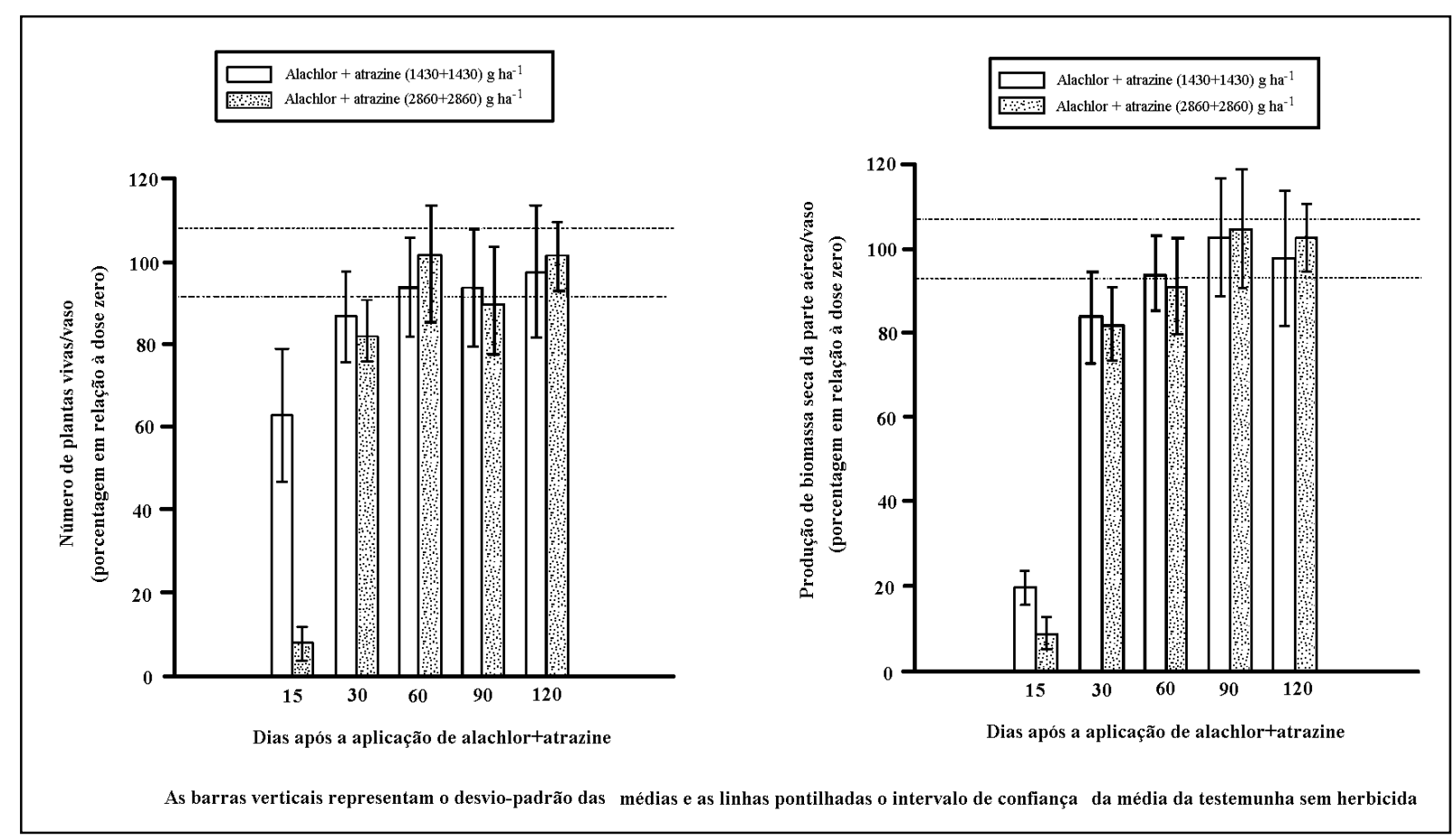

Figura 2 - Efeito de duas doses de de alachlor+atrazine sobre o número de plantas vivas e peso da massa seca da parte aérea das plantas de canola semeadas em amostras de solo coletadas em diferentes épocas após a aplicação dos herbicidas no campo. 
Os resultados obtidos confirmam a baixa tolerância da canola ao imazaquin. A dose de $150 \mathrm{~g}$ e.a. ha ${ }^{-1}$ aplicada em pré-emergência após a semeadura da cultura inibiu a germinação em $100 \%$, quando a semeadura foi feita em solo argiloso, $\mathrm{pH} 5,6$, com 2\% de matéria orgânica (OLIVEIRA JR., 1994). A análise do conjunto dessas informações sugere que, no caso da presença de resíduos de imazaquin no solo, se não houver a redução do estande da canola, dificilmente o crescimento e o desenvolvimento da cultura poderão ser afetados posteriormente.

Para alachlor+atrazine, observou-se que, embora os efeitos sobre a porcentagem de germinação e sobre o acúmulo de massa seca (Figura 2) tenham sido intensos nas amostras coletadas até os 30DAA, os sintomas de fitotoxicidade decresceram gradativamente e, a partir dos 90DAA, não se observou nenhum efeito negativo sobre as características avaliadas. Nas plantas que evidenciaram fitotoxicidade nas primeiras épocas de semeadura, os sintomas observados foram de clorose generalizada, progredindo à necrose e morte das plântulas.

A canola semeada nas áreas que receberam as aplicações dos herbicidas no campo (125DAA dos herbicidas) não evidenciou nenhum sintoma visual de fitotoxicidade, nem com relação à germinação, ao crescimento ou à produtividade, ratificando os resultados obtidos em casa de vegetação (Tabela 2).

\section{CONCLUSÕES}

Alachlor+atrazine e imazaquin, em pelo menos uma das doses utilizadas, afetaram a germinação da canola em amostras de solo coletadas

Tabela 2 - Estande final e produtividade da cultura da canola semeada em áreas cultivadas com soja e milho 125DAA de imazaquin e alachlor+atrazine, respectivamente. Os valores representam as médias dos experimentos conduzidos em Iguatemi e Cianorte, PR.

\begin{tabular}{|c|c|c|c|}
\hline $\begin{array}{c}\text { Cultura e herbicida } \\
\text { utilizado }\end{array}$ & Dose & $\begin{array}{c}\text { Estande final } \\
\left(\text { mil plantas } \mathrm{ha}^{-1}\right)\end{array}$ & $\begin{array}{l}\text { Produtividade de } \\
\text { grãos }\left(\mathrm{kg} \mathrm{ha}^{-1}\right)\end{array}$ \\
\hline \multirow[t]{3}{*}{ Soja - imazaquin } & 0g e.a. ha ${ }^{-1}$ & 382,5 & 3197 \\
\hline & 150 g e.a. ha ${ }^{-1}$ & 378,7 & 3330 \\
\hline & 300 g e.a. ha ${ }^{-1}$ & 391,3 & 3400 \\
\hline DMS $_{\text {soja }}$ & & $27,8^{\mathrm{ns}}$ & $357^{\text {ns }}$ \\
\hline \multirow[t]{3}{*}{ Milho - alachlor+atrazine } & $0 \mathrm{~g} \mathrm{ha}^{-1}$ & 53,4 & 4560 \\
\hline & $1430+1430 \mathrm{~g} \mathrm{ha}^{-1}$ & 54,2 & 4381 \\
\hline & $2860+2860 \mathrm{~g} \mathrm{ha}^{-1}$ & 54,1 & 4690 \\
\hline $\mathrm{DMS}_{\text {milho }}$ & & $4,2^{\mathrm{ns}}$ & $378^{\mathrm{ns}}$ \\
\hline
\end{tabular}

até 30 e 60DAA, respectivamente. No entanto, após esses períodos, não foram observados quaisquer efeitos sobre a germinação ou desenvolvimento da canola, mesmo em amostras provenientes de locais em que se aplicou o dobro da dose recomendada. Nas condições em que os experimentos foram desenvolvidos, a germinação e desenvolvimento da canola no campo, após os cultivos de soja e milho nos quais se utilizaram imazaquin e alachlor+atrazine, não foram afetados pela atividade residual desses herbicidas no solo.

\section{REFERÊNCIAS BIBLIOGRÁFICAS}

AHRENS, W.H. Herbicide handbook. 7.ed. Champaign : Weed Science Society of America, 1994. 352p.

BOWMAN, B.T. Mobility and persistence of the herbicides atrazine, metolachlor, and terbuthylazine in Plainfield sand determined using field lisimeters. Environmental Toxicology and Chemistry, v.8, p.485-491, 1989.

EMBRAPA. Empresa Brasileira de Pesquisa Agropecuária. Centro Nacional de Pesquisa de Soja, Londrina, PR. Recomendações técnicas para a cultura da soja no Paraná 1993/94. Londrina : EMBRAPA-CNPSo/OCEPAR, 1993. 128p. (EMBRAPA-CNPSo. Documentos, 62).

FLURY, M. Experimental evidence of transport of pesticides through field soils - a review. Journal of Environmental Quality, v.25, p.25-45, 1996.

JOHNSON, D.H., TALBERT, R.E. Cotton (Gossypium hirsutum) response to imazaquin and imazethapyr soil residues. Weed Science, v.44, p.156-161, 1996.

MELHORANÇA, A., CONSTANTIN, J., PEREIRA, F.A.R. Plantas daninhas. In: EMBRAPA Centro de Pesquisa Agropecuária do Oeste (Dourados, MS). Milho: informações técnicas . Dourados : EMBRAPA, 1997. p.111-132. (EMBRAPA-CPAO - Circular Técnica, 5).

NAKAGAWA, L.E., LUCHINI, L.C., MUSUMECI, M.R., et al. Comportamento da atrazina em solos brasileiros em condições de laboratório. Pesquisa Agropecuária Brasileira, v.30, p.471-476, 1995

NOVO, M.C.S.S., CRUZ, L.S.P., PEREIRA, J.C.V.N.A., et al. Persistência de imazaquin em latossolo roxo cultivado com soja. Planta Daninha, v.15, n.1, p.30-38, 1997.

OLIVEIRA JR., R.S. Susceptibilidade da cultura da canola (Brassica napus var oleifera) a herbicidas. Revista UNIMAR, v.16, p.301-315, 1994.

OLIVEIRA JR., R.S., BORGHI, C.H., ZOTARELLI, L., et al. Tolerância da cultura da canola a herbicidas aplicados ao solo. In: ENCONTRO ANUAL DE INICIAÇÃO CIENTÍFICA $\quad$ CNPq/UEPG/UEL/UEM, 3. Ponta Grossa, PR, 1994. Anais... - Ponta Grossa, UEPG, 1994. p.144. 210p. 
RENNER, K.A., MEGGIT, W.F., PENNER, D. Effect of soil $\mathrm{pH}$ on imazaquin and imazethapyr adsorption to soil and phytotoxycity to corn (Zea mays). Weed Science, v.36, p.7883, 1988.

SAEG - Sistema para análises estatísticas, versão 7.0. Viçosa : Fundação Arthur Bernardes, 1997. n.p.

SANTOS, H.P., LHAMEY, J.C.B., DIAS, J.C.A. Efeito do espaçamento e da densidade de semeadura sobre o comportamento agronômico da colza. Pesquisa Agropecuária Brasileira, v.25, p.701-707, 1990.

SEMINÁRIO ESTADUAL DE PESQUISA DA CANOLA, 1. 1993, Cascavel. Informações preliminares para o cultivo da canola no estado do Paraná em 1993. Cascavel: [s.n.], 1992. 6p.

SILVA, A.A., FEDATO, E., FERREIRA, F.A. Respostas das culturas de milho (Zea mays L.) e de feijão (Phaseolus vulgaris L.) a resíduos de flumioxazin e imazaquin em latossolo roxo distrófico. In: CONGRESSO BRASILEIRO DA CIÊNCIA DAS PLANTAS DANINHAS, 20, 1995, Florianópolis, Resumos... Florianópolis : EPAGRI, 1995. p.163-164. 456p.

SILVA, A.A., OLIVEIRA JR., R.S., CASTRO FILHO, J.E. Avaliação da atividade residual no solo de imazaquin e triflu- ralin através de bioensaios com milho. Acta Scientiarum, v.20, n.3, p.291-295, 1998.

SILVA, J.B., ARCHÂNGELO, E.R., DUARTE, N.F., $\boldsymbol{e} \boldsymbol{t} \boldsymbol{a}$. Controle pré-emergente de plantas daninhas na cultura da soja com mistura de imazaquin+flumioxazin e seu efeito residual sobre o milho em sucessão. In: CONGRESSO BRASILEIRO DA CIÊNCIA DAS PLANTAS DANINHAS, 21, 1997, Caxambu. Resumos... Viçosa : SBCPD, 1997. p.135. 482p.

SILVA, J.C.M., CONCEIÇÃO, O.A., NETO, S.B. Subsídios para um programa de culturas alternativas de inverno: Santa Catarina. Florianópolis : Comissão Estadual de Planejamento Agrícola - CEPA/SC, 1981. p.99-116.

VIDAL, R.A. Herbicidas: mecanismos de ação e resistência de plantas. Porto Alegre : Ed. do autor, 1997. 165p.

WALKER, A., MOON, Y.H., WELCH, S.J. Influence of temperature, soil moisture and soil characteristics on the persistence of alachlor. Pesticide Science, v.35, p.109$116,1992$.

WALSH, J.D., DEFELICE, M.S., SIMS, B.D. Soybean (Glycine max) herbicide carryover to grain and fiber crops. Weed Technology, v.7, p.625-632, 1993.

Ciência Rural, v. 31, n. 2, 2001. 